\title{
Investigación sobre la Seguridad y Protección Radiológica en procedimientos radiológicos dentales. Cuanto hay en Chile
}

\author{
Research on Safety and Radiological Protection in Dental \\ Radiological Procedures. How much is there in Chile
}

\section{Carlos Ubeda}

Estimado editor:

De acuerdo con el Comité Científico de Naciones Unidas sobre los Efectos de la Radiación Atómica (UNSCEAR), las exposiciones médicas a radiaciones ionizantes representan actualmente la segunda principal fuente de irradiación a la población mundial (United Nations Scientific Committee on the Effects of Atomic Radiation, 2008). Los procedimientos de radiología dental son el tipo más frecuente de estudio radiológico, y representan el $21 \%$ del total de exámenes a nivel mundial. La cifra anual de exámenes dentales se estima que es de 520 millones aproximadamente, y su frecuencia anual se halla entre menos de uno y más de 800 por cada 1000 habitantes. Las dosis individuales son pequeñas, pero las dosis colectivas no se pueden ignorar, debido a la gran cantidad de procedimientos que se realizan (European Commission, 2004).

Dado el escenario mundial anterior, bien vale la pena preguntarse ¿Cuál será la investigación chilena en torno a la Seguridad y Protección Radiológica en procedimientos radiológicos dentales desarrollada en los artículos publicados por la revista International Journal of Odontostomatology?.

Luego de haber revisado los 24 números disponibles de la revista en su página de Scielo Chile entre los años 2010-2017 (Scielo, 2018) y, buscando los títulos que se relacionaran con procedimientos de radiología dental en seres humanos, encontramos 4 artículos (Hidalgo \& San Pedro, 2010; Zaror et al., 2011; Bassi et al., 2014; Ardila \& Villa-Correa, 2015) que utilizaban técnicas radiológicas intra y extraorales (proyecciones periapicales, craneales, etc), 7 artículos que también requirieron técnicas radiológicas extraorales, específicamente ortopantomografías (Schilling et al., 2010; Neves et al., 2012; Kumar et al., 2013; De Conto et al., 2013; Garay \& Olate, 2013; Schlaepfer-Sales et al., 2015: Bizcar et al., 2015) y finalmente, 5 artículos que incorporaron procedimientos de tomografía computarizada, incluyendo el haz cónico (Vargas et al., 2011; Alves \& Figueiredo, 2012; Visconti et al., 2014; Peyneau et al., 2017; Hernández et al., 2017). Sin embargo, en ninguno de estos estudios se realiza un análisis profundo, sobre las ventajas de una u otra técnica radiológica, desde el punto de vista de la Seguridad y Protección Radiológica para el paciente y los trabajadores. En este sentido, comprender las diferentes tecnologías disponibles que utilizan radiaciones ionizantes para generar una imagen, conocer los principios básicos de la Protección Radiológica, establecer Programas de Garantía y Protección Radiológica, medir los niveles de radiación a pacientes y trabajadores, resultan investigaciones relevantes a la hora de avanzar en la formación y conocimiento integral de los Odontólogos en Chile.

\section{REFERENCIAS BIBLIOGRÁFICAS}

Alves, N. \& Figueiredo, D. N. Image of bilateral hypertrophy of the mandibular coronoid process through Cone-beam computed tomography. Int. J. Odontostomat., 6(2):225-8, 2012.

Ardila, M. C. M. \& Villa-Correa, Y. A. Gram-negative enteric rods associated to early implant failure and peri-implantitis: Case report and systematic literature review. Int. J. Odontostomat., 9(2):32936, 2015.

Bassi, A. P. F.; Gealh, W. C.; Pereira, C. C. S.; Jardim, E. C. G.; Filho, O. M. \& Garcia Junior, I. R. Radiographic and clinical evaluations of the zygomatico-maxillary complex fractures treated by 2.0 miniplates system. Int. J. Odontostomat., 8(1):77-83, 2014. 
Bizcar, M. B.; Sandoval, V. P. \& Navarro, C. P. Radiographic analysis and prevalence of impacted maxillary canine teeth in children between 8 and 16 years. Int. J. Odontostomat., 9(2):283-7, 2015.

De Conto, F.; De Bona, M.; Rui, G.; Rovani, G.; Rhoden, R. \& Flores, M. E. Maxillary sinusitis of odontogenic origin. surgical diagnosis and treatment. Int. J. Odontostomat., 7(3):421-6, 2013.

European Commission. Radiation Protection. European Guidelines on Radiation Protection in Dental Radiology. The Safe Use of Radiographs in Dental Practice. Issue No 136, Luxembourg, Directorate-General for Energy and Transport, Directorate $\mathrm{H}-$ Nuclear Safety and Safeguards, Unit H.4, 2004. Disponible en: https://ec.europa.eu/energy/sites/ener/files/documents/136_0.pdf

Garay, I. \& Olate, S. Currrent considerations in the study of image of soft tissue calcification in mandibular angle area. Int. J. Odontostomat., 7(3):455-64, 2013.

Hernández, V. S.; Donoso, Z. M.; Sanhueza, T. C.; Linco, O. J. \& Riquelme, C. S. Evaluation of periapical lesions by Cone Beam Computed Tomography in patients referred for apical surgery. Int. J. Odontostomat., 11(2):128-32, 2017.

Hidalgo, R. A. \& San Pedro, V. J. Relationship between lingual foramen and mental spines, and evaluation of lingual canal by using periapical and extra-oral radiographs. Int. J. Odontostomat., 4(3):295-302, 2010

Kumar, K.; George, G. B.; Padiyath, S. \& Rupak, S. Odontoameloblastoma: A diagnostic dilemma for maxillofacial radiologist. Int. J. Odontostomat., 7(2):203-6, 2013.

Neves, F. S.; Ramirez-Sotelo, L. R.; de Freitas, D. Q.; Haiter-Neto, F. \& Bóscolo, F. N. Bífid mandibular condyle: A diagnostic challenge. Int. J. Odontostomat., 6(3):327-30, 2012.

Peyneau, P. D.; da Costa, E. D.; Verner, F. S.; de Freitas, D. Q.; Almeida, S. M. \& Ambrosano, G. M. B. Cone Beam Computed Tomography in the diagnosis of dens invaginatus: Case report. Int. J. Odontostomat., 11(1):89-94, 2017.

Schilling, L. J.; Schilling, Q. A. \& San Pedro, V. J. Mandibular canal duplication prevalence, digital panoramic radiography analysis. Int. J. Odontostomat., 4(3):207-213, 2010.

Schlaepfer-Sales, C. B.; Valverde, L. F.; Dias, R. B.; Pimentel, B. N. A. S.; Melo, L. A.; Dos Santos, J. N.; Sarmento, V. A. \& Rocha, C. A. $\mathrm{G}$. Association between radiographic features and cell proliferation in keratocystic odontogenic tumor. Int. J. Odontostomat., 9(2):219$26,2015$.

Scielo. International Journal of Odontostomatology. Sitio Web, 2018. Disponible en: http://www.scielo.cl/scielo.php?script=sci_ issues \&pid=0718-381X\&lng=es\&nrm=iso

United Nations Scientific Committee on the Effects of Atomic Radiation. UNSCEAR 2008 Report Vol. I. Sources and Effects of lonizing Radiation. Viena, United Nations Scientific Committee on the Effects of Atomic Radiation, 2008. Disponible en: http:// www.unscear.org/unscear/en/publications/2008_1.html

Vargas, F. I.; Mayer, O. C.; Hervozo, S. P. \& Navia, G. E. Cementoossifying fibroma: Clinical, radiological and histological analysis of 2 cases in one family. Int. J. Odontostomat., 5(3):270-8, 2011.

Visconti, M. A. P. G.; Junqueira, R. B.; Verner, F. S.; Rodrigues, A. A. S.; Devito, K. L. \& Visconti Filho, R. F. Cone-beam computed tomography as complementary tool in diagnosis and surgical planning of dentigerous cyst: case report. Int. J. Odontostomat., 8(1):85-91, 2014.

Zaror, S. C.; Vergara, G. C.; Díaz, M. J. \& Aracena, R. D. Ferric sulfate and MTA pulpotomies in primary teeth: A case series. Int. J. Odontostomat., 5(1):77-82, 2011.
Dirección para correspondencia:

Carlos Ubeda de la Cerda, MSc, PhD.

Decano Facultad de Ciencias de la Salud

Universidad de Tarapacá

Arica

CHILE

Email: cubeda@uta.cl carlos.ubeda.uta2@gmail.com

Recibido : 27-01-2018

Aceptado : 13-04-2018 